\title{
Evaluación y monitoreo de la calidad ambiental del agua en el proyecto sistema de riego Canal N, provincia de Melgar-Puno, Perú
}

\author{
Evaluation and monitoring of the environmental quality of water in the project \\ irrigation system Canal N, province of Melgar - Puno, Peru
}

\author{
*Jesús Wiliam Huanca-Arohuanca \\ ${ }^{2}$ Sandra Beatriz Butrón Pinazo \\ ${ }^{2}$ Luis Alberto Supo Quispe \\ ${ }^{3}$ Felipe Supo Condori
}

\author{
ORCID: 0000-0002-7353-1166 \\ ORCID: 0000-0001-6653-8266 \\ ORCID: 0000-0001-7892-3460 \\ ORCID: 0000-0002-5007-9779
}

\section{RESUMEN}

La investigación tuvo como propósito realizar el monitoreo y evaluación de la calidad ambiental del agua en el proyecto "Mejoramiento y ampliación del servicio de agua para el sistema de riego Canal N, del distrito de Cupi, provincia de Melgar - Puno". En tal sentido, el proyecto ocasionó en el agua una perturbación, afectando la calidad ambiental de esta. Los resultados de los parámetros físico-químicos han evidenciado que el agua del río Llallimayo cumple con los Estándares de Calidad Ambiental - Categoría 3: "Riego de Vegetales y Bebida de Animales" (D.S.002 - 2008 MINAM), para los parámetros de temperatura, conductividad eléctrica, oxígeno disuelto y demanda bioquímica de oxígeno. Sin embargo, los parámetros correspondientes al $\mathrm{pH}$, aceites y grasas reflejaron una elevación en comparación con los Estándares de Calidad Ambiental, dando a entender que el pH se encuentra elevado debido a que en los meses de octubre, noviembre y diciembre empiezan las lluvias y precipitaciones. En cuanto a los aceites y grasas, estas se encuentran fuera del rango permitido debido a que, las actividades de mantenimiento de equipos y maquinarias ocasionan derrames de aceites y combustibles al cuerpo de agua.

Palabras clave: Aceites y grasas, agua, calidad ambiental, monitoreo, parámetros físico químicos.

\section{ABSTRACT}

The purpose of the research was to monitor and evaluate the environmental quality of water in the project "Improvement and expansion of the water service for the Canal N irrigation system, in the district of Cupi, Melgar Puno province". In this sense, the project caused a disturbance in the water, affecting the environmental quality of the water. The results of the physical-chemical parameters have shown that the water of the Llallimayo River complies with the Environmental Quality Standards - Category 3: "Vegetable Irrigation and Animal Drink" (D.S.002 - 2008 MINAM), for temperature parameters, electrical conductivity, dissolved oxygen and biochemical oxygen demand. However, the parameters corresponding to $\mathrm{pH}$, oils and fats reflected an elevation compared to the Environmental Quality Standards, implying that $\mathrm{pH}$ is high due to the beginning of rains and precipitation in October, November and December. As for oils and greases, they are outside the permitted range because the maintenance activities of equipment and machinery cause oil and fuel spills to the waterbody.

Keywords: Oils and greases, water, environmental quality, monitoring, chemical physical parameters.

\footnotetext{
Facultad de Ciencias Sociales. Universidad Nacional de San Agustín. Arequipa - Perú.

${ }^{2}$ Facultad de Ciencias Biológicas. Universidad Nacional del Altiplano. Puno - Perú.

${ }^{3}$ Facultad de Ciencias Sociales. Universidad Nacional del Altiplano. Puno - Perú.

* Autor de correspondencia: jhuancaar@unsa.edu.pe
} 


\section{INTRODUCCIÓN}

La disponibilidad de agua es elemental para la vida y el desenvolvimiento económico de cualquier región del mundo. Por ello, los recursos disponibles deben repartirse entre numerosos usuarios, teniendo en cuenta las necesidades del medio ambiente. Durante muchos años, todos los recursos eran considerados disponibles para cualquier uso antrópico, sin tener en consideración la calidad o las necesidades para los usos ambientales(A. Fernández, 2012).

Entonces, la construcción sostenible deberá entenderse como el desarrollo de la construcción tradicional, pero con una responsabilidad considerable con el medio ambiente por todas las partes y participantes. Lo que implica un interés creciente en todas las etapas de la construcción, considerando las diferentes alternativas en el proceso de edificación en favor de la minimización del agotamiento de los recursos, previniendo la degradación ambiental o los prejuicios para proporcionar un ambiente saludable (Gómez, 2014). Sin embargo, hoy en día, la mayor preocupación es que los procesos de consumo directo y las actividades humanas indirectas que utilizan el agua como recurso den origen a la contaminación (Peña, 2005). Por ejemplo, las actividades como vertido de agua residual doméstica, lavado de vehículos y acumulación de residuos sólidos que afectan la calidad del agua de las acequias(Romero, 2005).

Es por ello que, el Proyecto "Mejoramiento y ampliación del servicio de agua para el sistema de riego Canal N, provincia de Melgar-Puno" podría ocasionar una serie de perturbaciones al contexto natural. Asimismo, las actividades productivas y los servicios causarán impactos en el ambiente. Es así que, la actividad humana aparece como una causa significativa del cambio ambiental, principalmente como resultado del conflicto entre mantener y utilizar el medio, es decir, debido al desarrollo, explotación de recursos físicos, construcción, urbanización, cambio de uso de la tierra, y deposición de residuos. Esto a expensas de la integridad del componente biótico de los recursos ambientales y biológicos.

El recurso hídrico ha sido fuertemente afectado por sustancias cada vez más agresivas y difíciles de tratar debido a naturaleza química presentes en desperdicios que caen a las corrientes. Por tal razón, es importante evaluar el impacto del ser antropológico (Huanca-Arohuanca, 2019; Huanca-Arohuanca \& Canaza-Choque, 2019; Huanca-Arohuanca, 2020: Huanca-Arohuanca, Canaza-Choque, Escobar-Mamani \& Ruelas, 2020) sobre los recursos hídricos a través del estudio de la naturaleza química, física y biológica del agua, mediante programas de monitoreo (Samboni, Carbajal \& Escobar, 2007). Sin embargo, el tratamiento de los datos obtenidos en el monitoreo suele ser una tarea dispendiosa y en muchas ocasiones de difícil entendimiento para los diferentes actores involucrados en el proceso de la valoración de la calidad, pues, en la actualidad los valores obtenidos deben permitir resolver diferentes tipos de conflictos como el uso del agua y la integridad ecológica de los sistemas acuáticos, los cuales involucran también aspectos socioeconómicos (Fernández, Ramírez \& Solano, 2001)

Por otro lado, la importancia del impulso de un modelo de gestión, en el rubro de la construcción, radica en la conducción de los elementos y dificultades ambientales de un área determinada por los diferentes actores sociales, mediante el uso selectivo de herramientas de gestión ambiental, con la finalidad de prevenir y/o mitigar el impacto generado por las empresas constructoras en el entorno inmediato. Con esa especial dependencia es oportuno realizar un análisis de los problemas ambientales que se están dando y tratar de buscar soluciones adecuadas que ayuden a recuperar la calidad ambiental. Es importante señalar que, en la actualidad, la calidad ambiental del agua, suelo $y$, aire son factores importantes para la conservación del medio ambiente y la salud de los organismos vivientes. Bajo esa línea, Lara (2010) afirma que los principales impactos negativos en la fase de operación ocasionan riesgos en la calidad del agua; por lo tanto, existe la probabilidad de peligro en la salud humana. Por su parte, Guerrero (2014) menciona que las actividades de operación y mantenimiento en plantas de tratamiento se encuentran con niveles altos de grasas y aceites, debido al daño en el filtro ascendente y falta de mantenimiento.

Por tanto, en investigaciones locales existen datos sobre la calidad ambiental del agua. Por ejemplo, Lobato (2013) realizó el monitoreo de las aguas que discurren en el río Ramis, precisando que los 
impactos ambientales causaron la colmatación de los canales y tomas de riego, incidiendo negativamente en la producción agropecuaria y deteriorando la calidad del agua. Bajo la misma lógica, Cutire (2015) abordó los efectos que presenta la calidad del agua del río Llallimayo, por lo que los parámetros modificados durante la ejecución de la obra han tenido un análisis comparativo con las normativas legales ambientales tanto nacionales como internacionales.

En suma, la investigación tuvo como objetivo monitorear y evaluar la calidad ambiental del agua del proyecto "Mejoramiento y ampliación del servicio de agua para el sistema de riego Canal N en la provincia de Melgar-Puno".

\section{MATERIAL Y MÉTODOS}

\section{Monitoreo de la calidad del agua}

El monitoreo de calidad de agua se realizó en el área de influencia del proyecto "Mejoramiento y ampliación del servicio de agua del sistema de riego Canal N"; cuyos cuerpos de aguas muestreados se clasificaron en agua naturalsuperficiales-agua de río.

Tabla 1. Estaciones de monitoreo

\begin{tabular}{lclc}
\hline \multicolumn{1}{c}{ Tipo de Agua } & Estación & \multicolumn{1}{c}{ Descripción } & $\begin{array}{c}\text { Coordenadas (UTM) } \\
\text { m }\end{array}$ \\
\hline & PM-1 & $\begin{array}{l}\text { Río Llallimayo - a 100 } \\
\text { m aguas arriba de la }\end{array}$ & E:304 161 \\
$\begin{array}{l}\text { Agua natural- } \\
\text { superficiales-agua de }\end{array}$ & & $\begin{array}{l}\text { bocatoma } \\
\text { Río Llallimayo - a 100 }\end{array}$ & E:304 020 \\
río. & PM-2 & moguas abajo de la & N:8 354 258 \\
& & bocatoma & \\
\hline
\end{tabular}

Nota: Determinado con el Datum: World Geodetic System 1984.

\section{Parámetros de campo}

Los parámetros medidos fueron el $\mathrm{pH}$, la temperatura $\left({ }^{\circ} \mathrm{C}\right)$, la $\mathrm{CE}(\mu \mathrm{S} / \mathrm{cm})$ y el oxígeno disuelto con un multiparámetro Hanna, modelo H19828. Además, se tomaron muestras al azar en botellas de plástico y vidrio, para luego proceder a sellar, catalogar y preservar la muestra para su respectivo análisis

\section{Parámetros de ensayo en laboratorio}

Las muestras para el análisis fueron transportadas al laboratorio de calidad ambiental de la UANCV, las mismas que fueron rotuladas y conservadas de acuerdo con los protocolos establecidos. Luego, se realizaron los análisis para la $\mathrm{DBO}_{5}$ y para aceites y grasas. Por último, los métodos utilizados fueron tomados de los Standard Methods for the Examination of Water and Wastewater, 17thed, APHA-AWWA-WEF, 2005.

\section{Análisis estadístico}

El análisis estadístico se realizó con el Software Estadístico Statgraphics Centurion XVI. Por lo cual, se puso especial atención al aceite y la grasa debido a su escasa solubilidad en el agua y su tendencia a separarse de la fase acuosa, además de producir un impacto estético, reducen la reoxigenación, disminuyendo el oxígeno disuelto y absorbiendo la radiación solar, afectando a la actividad fotosintética. 


\section{RESULTADOS}

Monitoreo de la calidad del agua

Tabla 2. Resultados de los parámetros físico-químicos en los puntos de monitoreo

\begin{tabular}{|c|c|c|c|c|}
\hline \multicolumn{5}{|c|}{ Temperatura } \\
\hline MES DE MONITOREO & PM-1 & PM-2 & $\mathbf{E C A}^{\mathbf{1}}$ & $\mathbf{E C A}^{2}$ \\
\hline Octubre & 13.5 & 18.4 & NA & NA \\
\hline Noviembre & 12.7 & 16.3 & & \\
\hline Diciembre & 13.7 & 18.0 & & \\
\hline \multicolumn{5}{|c|}{ pH } \\
\hline Octubre & 8.50 & 8.40 & $6.5-8.5$ & $6.5-8.4$ \\
\hline Noviembre & 9.12 & 9.15 & & \\
\hline Diciembre & 9.10 & 9.12 & & \\
\hline \multicolumn{5}{|c|}{ Conductividad eléctrica } \\
\hline Octubre & 523 & 527 & $<2000$ & $\leq 5000$ \\
\hline Noviembre & 527 & 530 & & \\
\hline Diciembre & 522 & 525 & & \\
\hline \multicolumn{5}{|c|}{ Oxígeno disuelto } \\
\hline Octubre & 7.43 & 5.05 & $\geq 4$ & $\geq 5$ \\
\hline Noviembre & 7.49 & 5.08 & & \\
\hline Diciembre & 7.45 & 5.07 & & \\
\hline \multicolumn{5}{|c|}{ Demanda bioquímica de oxígeno (DBO5) } \\
\hline Octubre & 6.6 & 7.2 & 15 & $\leq 15$ \\
\hline Noviembre & 6.4 & 7.1 & & \\
\hline Diciembre & 6.5 & 7.1 & & \\
\hline \multicolumn{5}{|c|}{ Aceites y grasas } \\
\hline Octubre & 0.95 & 1.3 & 1 & 1 \\
\hline Noviembre & 0.95 & 1.1 & & \\
\hline Diciembre & 0.95 & 1.1 & & \\
\hline
\end{tabular}

(1) DS N ${ }^{\circ}$ 002-2008-MINAM. Estándares Nacionales de Calidad Ambiental para Agua. Categoría 3. Parámetros para riego de vegetales de tallo bajo y tallo alto.

(2) DS No 002-2008-MINAM. Estándares Nacionales de Calidad Ambiental para Agua. Categoría 3. Parámetros para bebidas animales.

NA: No Aplica.

En el punto de monitoreo 1 (aguas arriba de la bocatoma), estas fueron de $13.5^{\circ} \mathrm{C}, 12.7^{\circ} \mathrm{C}$ y 13.7 ${ }^{\circ} \mathrm{C}$. En el punto de monitoreo 2, las temperaturas fueron de $18.4{ }^{\circ} \mathrm{C}, 16.3{ }^{\circ} \mathrm{C}$ y $18.0^{\circ} \mathrm{C}$. De los resultados obtenidos sobre los niveles de temperatura durante los meses de octubre a diciembre, al comparar el punto de monitoreo 1 con el punto de monitoreo 2, se encontró que existe una diferencia, esto por las acciones antrópicas como los efectos de la deforestación, los vertidos industriales y la construcción. Al comparar los dos puntos de monitoreo con los Estándares Nacionales de Calidad Ambiental para Agua. Categoría 3: Parámetros para riego de vegetales tallo y para bebidas de animales (D.S. $\mathrm{N}^{\circ}$ 002-2008-MINAM), se observa que no aplican en ese parámetro los ECA para agua (Tabla 2).
Los niveles de $\mathrm{pH}$, en la fase de operación, que corresponden a los meses de octubre, noviembre y diciembre en el punto de monitoreo 1 (aguas arriba de la bocatoma), fueron de 8.50, 9.12 y 9.10; en el punto de monitoreo 2 fueron de 8.40, 9.15 y 9.12. Los resultados obtenidos indican que los niveles de $\mathrm{pH}$ en los meses de noviembre y diciembre en cuanto al punto de monitoreo 1 y 2 son diferentes, debido a que se presentaron lluvias en el mes de noviembre y se presume que las rocas existentes por la zona tienen mayor presencia de carbonatos. Motivo por el cual, se presentó un arrastre de carbonatos por las lluvias, generando así el aumento del $\mathrm{pH}$ en el río Llallimayo. En cuanto a los valores de $\mathrm{pH}$ registrados en los puntos evaluados en el mes de octubre se encontraron dentro del rango establecido. Sin embargo, los resultados de los meses de noviembre y diciembre no se encuentran dentro del rango establecido. 
Por otra parte, los niveles de conductividad eléctrica en el punto de monitoreo 1 en octubre, noviembre y diciembre fueron de 523,527 y 522 ; en el punto de monitoreo 2 fueron de 527,530 y 525. Los niveles de oxígeno disuelto en los meses de octubre, noviembre y diciembre en el punto de monitoreo 1 fueron de 7.43, 7.49 y 7.45; en el punto de monitoreo 2 fueron de 5.05, 5.08 y 5.07. Sobre los niveles de oxígeno disuelto, al comparar el punto de monitoreo 1 con el punto de monitoreo 2 , encontramos que existe diferencia, la disminución está en función a la presencia de materia orgánica oxidable.

Los niveles de $\mathrm{DBO}_{5}$ en los meses de octubre, noviembre y diciembre en el punto de monitoreo 1 fueron de 6.6, 6.4 y 6.5; en el punto de monitoreo 2 fueron de 7.2, 7.1 y 7.1. Es más, los niveles de $\mathrm{DBO}_{5}$ muestran una diferencia en cuanto al punto de monitoreo 1 y 2 , la demanda bioquímica de oxígeno es una medida de la cantidad de oxígeno utilizado por los microorganismos durante la estabilización de la materia orgánica biodegradable, bajo condiciones aerobias, en un período de 5 días y a $20{ }^{\circ} \mathrm{C}$. Siendo menores los valores registrados de $\mathrm{DBO}_{5}$ en los puntos evaluados; cumpliendo, por lo tanto, con el valor establecido en los Estándares Nacionales de Calidad Ambiental para Agua, Categoría 3: Parámetros para riego de vegetales tallo y para bebidas de animales (D.S. N ${ }^{\circ}$ 002-2008MINAM).

En cuanto a los niveles de aceites y grasas durante los meses de octubre, noviembre y diciembre en el punto de monitoreo 1, estos fueron de 0.95 (para loa tres meses) y en el punto de monitoreo 2 fueron de 1.3, 1.1 y 1.1. Es que, los niveles de aceites y grasas muestran una diferencia entre el punto de monitoreo 1 y 2 . De los resultados obtenidos se puede notar que los niveles de aceites y grasas, en el punto de monitoreo 2, se encontraron fuera de los rangos establecidos en los Estándares Nacionales de Calidad Ambiental para Agua (D.S. No 002-2008-MINAM). Esto debido a que el agua se encuentra con restos de aceites y grasas provenientes de los equipos y maquinarias del proyecto.

Tabla 3. Análisis estadístico para aceites y grasas

\begin{tabular}{lcc}
\multicolumn{1}{c}{ Categorías } & PM-1 & PM-2 \\
\hline Recuento & 4 & 4 \\
Promedio & 0.95 & 1.1125 \\
Desviación Estándar & 0 & 0.143614 \\
Coeficiente de Variación & $0 \%$ & $12.9091 \%$ \\
Mínimo & 0.95 & 0.95 \\
Máximo & 0.95 & 1.3 \\
Rango & 0 & 0.35 \\
Desviación Estándar & 0 & 0.143614 \\
Varianza & 0 & 0.020625 \\
Gl & 3 & 3 \\
Intervalos de confianza del 95.0_\% & & \\
Desviación Estándar de PM-1: [0; 0] & & \\
Desviación Estándar de PM-2: & & \\
[0.0813562; 0.535474] & & \\
Prueba-F para comparar Desviaciones Estándar & \\
valor-P = 0 & & \\
alfa = 0.05. & & \\
\hline
\end{tabular}

Se realizó el análisis estadístico solo para aceites y grasas, debido a que este parámetro se encontró por encima de los márgenes establecidos en los ECA. Entonces, para la determinación de los resultados, se partirá de la premisa que cuando $\mathrm{p}$ es menor que $\alpha$ (nivel de significancia asumido que es de $5 \%$ ).

\section{DISCUSIÓN}

Los resultados difieren de Cutire (2015), quien reportó que los niveles de temperatura en las aguas arriba de la bocatoma, dentro de la fase de ejecución que corresponde al mes de septiembre, fueron de $11.2^{\circ} \mathrm{C}$ y $13.1{ }^{\circ} \mathrm{C}$; y en aguas debajo de la bocatoma fue de $18.2^{\circ} \mathrm{C}$. Mientras que 
Sinokrot \& Stefan (1993), refieren que la predicción exacta de la temperatura del agua ha adquirido de nuevo gran interés debido a la amenaza del cambio global, además que es necesario un análisis más dinámico y determinístico para predecir los efectos de las actividades del hombre en la cuenca de un río. Según Barthem (2003), se espera que aumenten los valores de temperatura en los ríos (no en las pequeñas quebradas con cobertura vegetal), en la época de estiaje, acarreando una bajada en los niveles de oxígeno disuelto. Sin embargo, Echarri (1998) menciona que la temperatura óptima del agua para beber se encuentra entre los $10^{\circ} \mathrm{C}$ y los $15{ }^{\circ} \mathrm{C}$. Por tanto, el aumento de temperatura disminuye la solubilidad de gases (como el oxígeno) y aumenta, en general, la de las sales.

SegúnCutire (2015), el pH es ligeramente alcalino debido a las formaciones geológicas predominantes (rocas calizas) en el área del proyecto; encontrando que los niveles de $\mathrm{pH}$, en la fase de ejecución que obedece al mes de septiembre. En el punto 1 fueron de 8.16 y 8.54, y en el punto 2 fue de 8.51 . El pH del agua es una característica muy importante, por ser un factor que afecta el equilibrio entre la mayoría de las especies químicas, el potencial corrosivo del agua, la conveniencia del agua para mantener los organismos vivientes, y la mayoría de las demás características de la calidad del agua. En general, se usa para expresar la intensidad de la condición ácida o alcalina existente en un cuerpo de agua. Los valores de $\mathrm{pH}$ dependen de las sales y los iones disueltos del agua, que esta va adquiriendo a lo largo de su recorrido en las cabeceras de la cuenca-(Galvis et al., 2006).

La época de avenida, según Calvo-Brenes y Mora (2007), puede alterar el $\mathrm{pH}$ de las aguas superficiales, debido a la introducción de una mayor carga contaminante en el río, producto de la erosión de los suelos. En tal sentido, para Gallardo-Mayenco, Macías y Toja (2004), el pH del agua está relacionado con la carga orgánica, y la alcalinidad muy determinada por la materia es la variable físico-química más correlacionada como indicadora de contaminación orgánica. Por tanto, el pH se va modificando debido a las descargas de efluentes agrícolas domésticos.

En cuanto a la conductividad eléctrica, Cutire (2015) considera que los niveles de las mismas, antes y en la fase de ejecución del proyecto, fueron de $525,0.44$ y 0.43 . Estos resultados difieren con los obtenidos en la investigación, presumiéndose que hubo un error en la lectura. La conductividad del agua depende de la concentración total de sustancias disueltas ionizadas en el agua y de la temperatura a la cual se haga la determinación. Por ello, cualquier cambio en la cantidad de sustancias disueltas, en la movilidad de los iones disueltos y en su valencia, implica un cambio en la conductividad. Así mismo, se correlaciona un aumento de la conductividad con un deterioro en las aguas con mayor cantidad de sólidos en suspensión; sin embargo, la geología de la cuenca juega un papel importante y determina gran parte de la carga iónica(Araujo, 2011). Y los valores de conductividad eléctrica dependerán de las sales y los iones disueltos del agua, que estas van adquiriendo a lo largo de su recorrido en las cabeceras de la cuenca-(Galvis et al., 2006).

Los niveles de oxígeno disuelto reportados por Cutire (2015) fue de 7.44, mostrando de esa manera la importancia del oxígeno en el agua es vital para la vida acuática (peces, plantas, bacterias aerobias, etc.), por ello la falta del mismo es dañina para ella. Así mismo, la falta de oxígeno disuelto es un indicador de contaminación que puede estar en función de la presencia de plantas acuáticas, materia orgánica oxidable, de organismos y de gérmenes aerobios, existencias de grasas, de hidrocarburos, de detergentes, etc. (Senamhi, 2007). El oxígeno molecular disuelto $\left(\mathrm{O}_{2}\right)$ es el más importante agente oxidante presente en las aguas naturales. Su determinación es vital por ser el factor que define la existencia de condiciones aerobias o anaerobias, además del grado de contaminación que pueda existir en estas aguas. La mayor parte de oxígeno proviene de la atmósfera y, en menor cantidad, de la acción fotosintética de las algas. En cambio, -McKinsey y Chapman (1998) indican que el oxígeno se encuentra inversamente relacionado a la temperatura, de tal forma que un aumento en la temperatura del agua implica una bajada de oxígeno, ya que lo acerca más a su punto de saturación favoreciendo su dilución y escape al aire.

$\mathrm{La} \mathrm{DBO}_{5}$ fue de 35 y 6.5 para el punto 1 y $7.0 \mathrm{en} \mathrm{el}$ punto 2. Con respecto al mes de agosto, se observa que este nivel de $\mathrm{DBO}_{5}$ difiere de los otros meses de monitoreo, presumiéndose que hubo un error al realizar la lectura. La demanda bioquímica de oxígeno es una medida de la cantidad de oxígeno utilizado por los microorganismos durante la 
estabilización de la materia orgánica biodegradable, bajo condiciones aerobias, en un período de 5 días y a $20^{\circ} \mathrm{C}$. La prueba de $\mathrm{DBO}$ es una de las más importantes en las operaciones de control de la contaminación de las corrientes; además de establecer los criterios de regulación, y para realizar estudios que evalúan la capacidad de purificación de cuerpos de agua receptores. Según Moran, Denisse, Gómez y Vicente (2009), la DBO es afectada por la temperatura del medio, por las clases de microorganismos presentes, por la cantidad y tipo de elementos nutritivos presentes; mostrando al final que el aumento de DBO puede indicar una mala calidad de agua.

En esa línea, Cutire (2015) menciona que los niveles de aceites y grasas, fueron de 0.95 en ambos puntos de monitoreo. La concentración de aceites y grasas interfieren con el intercambio de gases entre el agua y la atmósfera; además, no permiten el libre paso del oxígeno hacia el agua, ni la salida del $\mathrm{CO}_{2}$ del agua hacia la atmósfera. En casos extremos, pueden llegar a producir la acidificación del agua, el hecho de que sean menos densos que el agua e inmiscibles con ella hace que se difundan por la superficie, de modo que, las pequeñas cantidades de grasas y aceites pueden cubrir grandes superficies de agua; encareciendo los tratamientos de depuración, y algunos aceites, especialmente los minerales, suelen ser tóxicos. En tal sentido, su presencia complica el transporte de los residuos por las tuberías, su eliminación en unidades de tratamiento biológico y su disposición en las aguas receptoras(Patiño et al., 2002).

Los aceites y grasas se definen como el material orgánico, soluble en un solvente no polar, recuperado en una muestra dada (CrombetGrillet, Pérez-Pompa, Ábalos-Rodríguez \& Rodríguez-Pérez, 2013). Los aceites y grasas en el agua impiden el paso de la luz y el oxígeno y, por lo tanto, perjudican el desarrollo de microorganismos y la autodepuración (Sánchez, Rico \& Galvañ, 2020). En condiciones naturales, las aguas superficiales no deberían presentar contenidos de grasas y aceites. Sin embargo, se utilizan como indicador para medir el grado de contaminación por usos industriales y humanos, es decir, las fuentes de la concentración de grasas $\mathrm{y}$ aceites registrada en las aguas superficiales pueden deberse al lavado de vehículos pesados, livianos y motos(Urrutia, 2019).

Según -Ramos (2016), los aceites y grasas en los vertidos líquidos ocasionan dos tipos de preocupación a la hora de la depuración de las aguas residuales. Primero, reducen la mojabilidad de los sólidos en suspensión, evitando con ello su sedimentación. Y, en segundo lugar, reducen la formación de una cutícula que recubre los microorganismos encargados de la biodegradación, impidiendo con ello la captación de oxígeno por los mismos y disminuye su poder depurador. Por lo que Cabrera (2001) afirma que las concentraciones de grasas, aceites y residuos inorgánicos, hacen que las características físicas, químicas y biológicas de las aguas se vean alteradas, manifestándose cambios en su calidad y en el deterioro del paisaje.

En definitiva, Vallejo (2010) menciona que la presencia de aceites y grasas son perjudiciales para la vida acuática, porque el hecho de que sean menos densos que el agua e inmiscibles con ella, provoca que se forman membranas que se difunden sobre la superficie, De modo que, pequeñas cantidades de grasas y aceites pueden cubrir grandes superficies de agua. Además de producir un impacto estético, reducen la aeración y disminuyen la penetración de la luz solar necesaria para la fotosíntesis (producción primaria) de las plantas acuáticas.

\section{CONCLUSIONES}

Los resultados de los parámetros físico-químicos han evidenciado que el agua del río Llallimayo, cumple con los ECA - Cat. III: "Riego de vegetales y bebida de animales" (D.S. 002-2008 MINAM), dentro de los parámetros de temperatura, conductividad eléctrica, oxígeno disuelto y demanda bioquímica de oxígeno.

En primer término, en el parámetro $\mathrm{pH}$, se puede notar que refleja una ligera elevación en comparación con los ECA. Aquello permite conocer que el $\mathrm{pH}$ se encuentra elevado debido a que en esos meses empiezan las lluvias y precipitaciones.

En segundo término, los aceites y grasas se encuentran fuera del rango permitido en los meses de octubre a diciembre. Esto debido a que, en el proyecto, en la fase de operación en las actividades de mantenimiento de equipos y maquinarias, existen derrames de aceites y combustibles accidentales al cuerpo de agua.

Finalmente, para que lo efectos negativos se reviertan, es necesario que se tomen medidas preventivas y correctivas, ya que este parámetro no cumple con los ECA - Cat. III: "Riego de vegetales y bebida de animales". 


\section{REFERENCIAS}

Araujo, J. (2011). Proyecto hidrobiológico río Tahuamanu - Madre de Dios - Perú. Universidad de Florida.

Barthem, R. (2003). Aquatic Ecology of the Rio Madre de Dios: Scientific Bases for AndesAmazon Headwaters Conservation. Asociación para la Conservación de la Cuenca Amazónica (ACCA). Recuperado de: https://books.google.com.pe/books/about/Aqua tic Ecology of the Rio Madre de Dios.html ? id=hM-OmAEACAAJ\&redir esc $=\mathrm{y}$

Cabrera, C. (2001). Contaminación e impacto ambiental en la bahía de Chancay. Revista Del Instituto de Investigación, 4(8), 37-46. Recuperado de: http://sisbib.unmsm.edu.pe/bibvirtual/publicaci ones/geologia/v04_n8/contaminación_impacto ambiental.htm

Calvo-Brenes, G., \& Mora, J. (2007). Evaluación y clasificación preliminar de la calidad del agua de la cuenca del río Tárcoles y el Reventazón Parte I: Análisis de la contaminación de cuatro ríos del área metropolitana. Tecnología En Marcha, 20(2), 3-9.

Crombet-Grillet, S., Pérez-Pompa, N., ÁbalosRodríguez, A., \& RodríguezPérez, S. (2013). Caracterización de las aguas residuales de la comunidad "Antonio Maceo" de la Universidad de Oriente. Revista Cubana de Química, 25(2), 134-142. Recuperado de: https://www.redalyc.org/articulo.oa?id=44354 3735003

Cutire, M. (2015). Monitoreo de calidad de agua del Rio Llallimayo, distrito de Cupi-Melgar. Universidad Privada San Carlos.

Echarri, L. (1998). Ciencias de la Tierra y del Medio Ambiente. Teide. Recuperado de: http://www.tecnun.es/Asignaturas/Ecologia/Hi pertexto/Principal.html

Fernández, A. (2012). El agua: un recurso esencial. Revista QuímicaViva, 3, 147-170.

Fernández, N., Ramírez, A., \& Solano, F. (2001). Índices fisicoquímicos de la calidad del agua. Un estudio comparativo. Conferencia Internacional Usos Múltiples Del Agua: Para La Vida y El Desarrollo Sostenible, 81(3), 211$219 . \quad$ Recuperado de: https://www.academia.edu/1662411/Indices Fi sicoquimicos_de_Calidad_del_AguaUn Estudio Comparativo

Lobato, ${ }^{-}$A. (2013). Evaluación ambiental y programas de remediación de la cuenca alta del río Ramis (tesis de maestría). Universidad Nacional de Ingeniería, Perú.
Gallardo-Mayenco, A., Macías, S., \& Toja, J. (2004). Efectos de la descarga en la calidad del agua a lo largo de un río mediterráneo: el río Guadaira (Sevilla). Limnetica, 23(1-2), : 65-78. Recuperado de: https://www.limnetica.com/documentos/limnet ica/limnetica-23-1-p-65.pdf

Galvis, G., Mojica, J. I., Duque, S. R., Castellanos, C., Sánchez-Duarte, P., Arce, M., Gutiérrez, Á., Jiménez, L. F., Santos, M., Vejarano, S., Arbeláez, F., Prieto, E., \& Mauricio Leiva. (2006). Peces del medio Amazonas - Región de Leticia. In E. Panamericana (Ed.), Serie de Guias Tropicales de Campo No 5. Conservation Internacional (pp. 1-548). Formas e Impresos. doi: 10.1590/S1679-62252006000400011

Gómez, D. (2014). Recuperación de espacios degradados (2a ed.). Mundi-Prensa.

Guerrero, M. (2014). Estudio del impacto ambiental $y$ plan de manejo ambiental de la planta de tratamiento de aguas servidas de la junta administradora de agua potable $y$ alcantarillado de la Parroquia Quinchicoto (tesis de maestría). Universidad Técnica de Ambato, Ecuador.

Huanca-Arohuanca, J. W. (2019). El discurso filosófico y la violencia política en la Nación Aymara - Ácora (tesis de pregrado). Universidad Nacional del Altiplano, Perú. Recuperado de: http://repositorio.unap.edu.pe/handle/UNAP/1 2758

Huanca-Arohuanca, J. W. (2020). Contrahegemonía y la lucha por la educación en el sur del Perú. Editorial Académica Española.

Huanca-Arohuanca, J. W., \& Canaza-Choque, F. A. (2019). Puno: Educación rural y pensamiento crítico. Hacia una educación inclusiva. Revista Helios, 3(1), 97-108. doi: 10.22497/Helios.31.3106

Huanca-Arohuanca, J. W., Canaza-Choque, F. A., Escobar-Mamani, F., \& Ruelas, D. (2020). En defensa del pluralismo latinoamericano: las esferas de la justicia y la igualdad compleja en Michael Walzer. Un dilema pendiente por atender. Revista Chakiñan, 11. doi: 10.37135/chk.002.11.07

Lara, D. (2010). Estudio de impacto ambiental expost y formulación de un plan de manejo ambiental para el botadero de basura de el Ángel (tesis de pregrado). Universidad Técnica del Norte, Ecuador. Recuperado de: http://repositorio.utn.edu.ec/handle/123456789 $/ 115$ 
McKinsey, D., \& Chapman, L. (1998). Distribución de oxígeno disuelto y pescado en una primavera de Florida. Biología Ambiental de Peces, 53, 211-223. doi: 10.1023/A:1007497503542

Moran, F., Denisse, N., Gómez, C., \& Vicente, J. (2009). Proyecto investigación 2009 - demanda bioquímica de oxigeno [DSpace en ESPOL]. Recuerado de: https://www.dspace.espol.edu.ec/handle/12345 $6789 / 6165$

Patiño, P., Holguín, J., Barba, H., Cruz, C., Ramírez, C., Duque, A., \& Baena, L. (2002). Metodología para la adaptación de un índice de calidad del agua a las condiciones medio ambientales del río Cauca en el tramo Salvajina - La Virginia. Seminario Internacional: Visión Integral En El Mejoramiento de La Calidad Del Agua.

Peña, E. J. (2005). Algas como indicadoras de contaminación. U. del Valle. Recuperado de: https://www.libreriadelau.com/algas-comoindicadoras-de-contaminacion-u-del-valle9586704688-biologia/p

Ramos, V. K. (2016). Análisis de los parámetros fisicoquímicos de las aguas superficiales contaminadas en el estuario del rio chillón AAHH Márquez Callao 2016 (tesis de pregrado). Universidad Cesar Vallejo, Perú. Recuperado de: http://repositorio.ucv.edu.pe/handle/20.500.12 $692 / 40774$

Romero, B. M. (2005). Influencia de la actividad antropogénica en la calidad de las aguas de las acequias Cois, Yortuque y Pulen (tesis de doctorado). Universidad Nacional Pedro Ruiz Gallo, Perú.

Samboni, N. E., Carvajal, Y., \& Escobar, J. C. (2007). Revisión de parámetros fisicoquímicos comoRevisión de parámetros fisicoquímicos como indicadores de calidad y contaminación del agua indicadores de calidad y contaminación del agua. Revista Ingeniería e Investigación, 27(3), 172-181.

Sánchez, A. C. Y., Rico, D. P., \& Galvañ, P. J. V. (2020). Caracterización físico-química de las aguas residuales de la industria de curtición. Ingeniería Química, 403, 130-138.

Senamhi. (2007). Monitoreo de la calidad de agua de los ríos del Perú. Servicio Nacional de Metereología e Hidrología.

Sinokrot, B. A., \& Stefan, H. G. (1993). Stream temperature dynamics: Measurements and modeling. Water Resources Research, 29(7). doi: 10.1029/93WR00540

Urrutia, L. (2019). Estudio Físico-Químico y Bacteriológico de las aguas superficiales de la microcuenca río Siuna-RACCN (tesis de pregrado). Universidad Nacional Autónoma de Nicaragua. Recuperado de: https://repositorio.unan.edu.ni/10404/1/99212. pdf

Vallejo, A. A. (2010). Niveles de contaminación en el litoral sur de la bahía de Talara por aceitesgrasas y metales pesados (tesis de maestría). Universidad de Piura, Perú. Recuperado de: https://pdfs.semanticscholar.org/1897/5417b4c a1 edf74bb69b47564f44df14521fa.pdf 